\title{
Steady-shear rheological properties of model compatibilized blends
}

\author{
S. Velankar, ${ }^{\text {a) }}$ P. Van Puyvelde, J. Mewis, and P. Moldenaers \\ Department of Chemical Engineering, Katholieke Universiteit Leuven, \\ W. De Croylaan 46, B-3001 Leuven, Belgium
}

(Received 18 June 2003; final revision received 28 April 2004)

\begin{abstract}
Synopsis
Block copolymers may be added as surface-active compatibilizers in order to control the morphology of blends of immiscible polymers. The effects of such added compatibilizers on the rheological properties of droplet-matrix blends are investigated experimentally. Model blends composed of polyisobutylene (PIB) droplets in a polydimethylsiloxane (PDMS) matrix, compatibilized with a diblock copolymer of PIB and PDMS, are studied here. The viscosity ratio of the blends, i.e., the ratio of the viscosity of the droplets to that of the matrix, is varied from 0.1 to 2.7. The viscosity and the first normal stress difference under steady shear conditions, and complex moduli after cessation of shear are measured. It is found that addition of the compatibilizer slightly raises the magnitude of the terminal complex viscosity of blends at all ratios of viscosity. Furthermore, with addition of the compatibilizer, the terminal relaxation time is found to increase sharply at high viscosity ratios, whereas the steady shear $N_{1}$ is found to increase at low viscosity ratios. These experimental observations are consistent with Marangoni stress caused by flow-induced gradients in the compatibilizer concentration on the surface of compatibilized drops. It is shown that, qualitatively, the effects of the Marangoni stress are somewhat analogous to an increase in drop viscosity. (C) 2004 The Society of Rheology. [DOI: 10.1122/1.1765662]
\end{abstract}

\section{INTRODUCTION}

Blending of immiscible polymers is often the most economical means of achieving desired product properties. The processing history strongly affects the morphology and properties of a blend, hence, the relationship between flow and the structure of immiscible blends is a topic of intense current interest. Past research has elucidated the key role played by interfacial tension, and the volume fraction and relative viscosity of the components, and has yielded insight into the various phenomena that determine the flowinduced structural evolution of immiscible blends. Key features of the dynamics of immiscible blends as reviewed by Tucker and Moldenaers (2002) are as follows: For droplet-matrix morphologies, structural evolution during shear flow occurs primarily by the deformation, breakup, and coalescence of droplets. Droplet deformation is governed by two dimensionless numbers: the viscosity ratio, $p=\eta_{d} / \eta_{m}$, and the capillary number,

$$
C a=\frac{\text { deforming hydrodynamic stress }}{\text { restoring interfacial stress }}=\frac{\dot{\gamma} \eta_{\mathrm{m}}}{\alpha / R}=\frac{R \dot{\gamma} \eta_{m}}{\alpha},
$$

\footnotetext{
a) Author to whom correspondence should be addressed; present address: Department of Chemical Engineering, University of Pittsburgh, Pittsburgh, PA 15261; electronic mail: velankar@pitt.edu
} 
where $R$ is the radius of the droplet, $\alpha$ is the interfacial tension, $\dot{\gamma}$ is the shear rate, and $\eta_{d}$ and $\eta_{m}$ are the viscosities of the droplet and matrix fluids, respectively. Such droplet deformation corresponds to storage of mechanical energy via interfacial deformation and confers viscoelasticity upon a blend. If the deforming stress is sufficiently high, i.e., if

$$
C a \geqslant C a_{\mathrm{cr}}(p),
$$

droplets breakup, primarily by simple rupture of moderately elongated droplets or capillary breakup of highly elongated fibrils. Here $C a_{\mathrm{cr}}$ is the critical capillary number for the breakup of droplets. Finally, flow induces collisions, and hence coalescence, of droplets [Chesters (1991)]. The capability to break up and coalesce implies that immiscible blends have no intrinsic length scale; the characteristic length scale of the blend, i.e., the drop size, depends on the flow field applied [Vinckier et al. (1996); Kitade et al. (1997)].

Surface-active species are often employed to promote blending of incompatible liquids. In the case of immiscible polymer blends, this "compatibilizer" may be added prior to blending or be generated by an interfacial chemical reaction during blending. Various aspects of the compatibilization of polymers have been discussed in recent reviews [di Lorenzio and Frigione (1997); Koning et al. (1998)]. Compatibilizers can reduce the interfacial tension between the immiscible phases of a blend, and thereby reduce the average drop size by facilitating breakup of droplets as per Eqs. (1) and (2). Reduction of interfacial tension is however not the only effect of compatibilizer addition [Van Puyvelde et al. (2001)]. Compatibilizers can also suppress coalescence of droplets [Sundararaj and Macosko (1995); Macosko et al. (1996); Milner and Xi (1996); Ramic et al. (2000); Velankar et al. (2001a); Van Hemelrijck et al. (2004)], induce interfacial viscoelasticity [Riemann et al. (1997); Jacobs et al. (1999); Van Hemelrijck et al. (2004)], and cause effects related to gradients in the interfacial concentration of the compatibilizer [de Bruijn (1993); Hu et al. (2000); Velankar et al. (2001b)].

Much work has been done on the morphological characteristics of compatibilized blends. Much less is known about their rheological properties [see Riemann et al. (1997); Iza et al. (2001); Zarraga et al. (2001); Sung et al. (2003), and references cited therein]. Most of the work has been performed with blends of commercial polymers whose complex rheological properties make it difficult to isolate the effects of the compatibilizer. Moreover, the amounts of compatibilizer added are often very large, generally more than $1 \%$ of the total and often as much as $10 \%$ of the total volume. These volume fractions are sufficiently large that the compatibilizer might form complex phases with the homopolymers or a separate phase of its own, and/or modify the rheological properties of the bulk phases of the blend significantly. A systematic study of the dynamic effects of a small amount of added compatibilizer on blends of simple fluids is still lacking. Here we investigate the rheological properties of blends of rheologically simple (i.e., nearly Newtonian) liquids, compatibilized by small amounts (1\% or less) of a well-characterized diblock copolymer in simple shear flow.

The blend system is composed of polyisobutylene (PIB) and polydimethylsiloxane (PDMS) compatibilized by an unentangled PIB-PDMS diblock copolymer. This system is an extension of past research on uncompatibilized PIB/PDMS blends [see Guido et al. (1999), and references therein], which are regarded as experimentally convenient substitutes for commercial polymer blends due to their low viscosity at room temperature, high immiscibility, nearly Newtonian behavior of the components, and transparency. In a previous article [Velankar et al. (2001b)], PIB/PDMS blends with viscosity ratio $p$ $\approx 1,10 \%$ of dispersed phase, and various (low) levels of compatibilizer were studied. The main observation in the previous study at $p \approx 1$ was that addition of a compatibilizer caused the steady-shear capillary number to increase to values well above $C a_{\mathrm{cr}}$ for 
TABLE I. Viscosity ratios and constituent of blends.

\begin{tabular}{|c|c|c|c|c|c|c|}
\hline $\begin{array}{l}\text { Blend } \\
\text { series }^{\mathrm{a}}\end{array}$ & $\begin{array}{c}T \\
\left({ }^{\circ} \mathrm{C}\right)\end{array}$ & $p$ & Drop $^{b}$ & $\begin{array}{c}\eta_{d} \\
(\mathrm{~Pa} \mathrm{~s})\end{array}$ & Matrix $^{c}$ & $\begin{array}{c}\eta_{m} \\
\text { (Pa s) }\end{array}$ \\
\hline B0.1-y & 32 & 0.10 & P950 & 18 & D200 & 181 \\
\hline B0.3-y & 32 & 0.29 & P1300 & 53 & D200 & 181 \\
\hline B $0.5-y$ & 23 & 0.54 & P1300 & 113 & D200 & 211 \\
\hline B1.1-y & 23 & 1.06 & P1300 & 113 & D100 & 107 \\
\hline B1.7-y & 17 & 1.74 & P1300 & 210 & D100 & 121 \\
\hline B2.7-y & 11 & 2.65 & P1300 & 366 & D100 & 138 \\
\hline
\end{tabular}

$a_{y}$ is the amount of compatibilizer in wt \% of dispersed phase and it can be 0,2 , or 10 .

${ }^{\mathrm{b}} \mathrm{P} 950=$ PIB with $\overline{M_{w}}=950 ; \mathrm{P} 1300=\mathrm{PIB}$ with $\overline{M_{w}}=1300$.

${ }^{\mathrm{c}} \mathrm{D} 200=$ Rhodorsil v. 200 000; D100 = Rhodorsil v. 100000.

breakup of uncompatibilized droplets [Velankar et al. (2001b)]. This suggests that the hydrodynamic stress required to break compatibilized droplets is higher than that expected from their interfacial tension. Based on earlier simulations [Stone and Leal (1990); $\mathrm{Li}$ and Pozrikidis (1997)], it was concluded that the most likely cause of this is a gradient in the interfacial concentration of the diblock copolymer along the drop's surface. This is believed to cause a gradient in interfacial tension (Marangoni stress), which resists deformation and breakup of compatibilized droplets.

In this paper, the effect of addition of a compatibilizer on the rheological properties of blends is investigated. Both the linear dynamic mechanical properties as well as the nonlinear steady-shear properties are discussed, with emphasis on compatibilizer effects at different viscosity ratios. As in the previous article [Velankar et al. (2001b)], the focus is not on the effect of added compatibilizer on the interfacial tension or the absolute size of droplets, but on rheological characteristics such as the relaxation time, viscosity, and normal stress. We ask, "Given a blend of certain viscosity ratio and volume fraction, how does a surface-active compatibilizer change the rheological properties?" As before [Velankar et al. (2001b)], in discussing the results, the compatibilizer is regarded only as a surfactant that decreases interfacial tension. Issues such as swelling of the blocks of the diblock by homopolymers or finite interfacial thickness are not discussed here.

\section{EXPERIMENT}

The blends were composed of two different grades of PIB (Parapol 950 and Parapol 1300, Exxon Chemical Co.) and two grades of PDMS (Rhodorsil v. 100000 and Rhodorsil v. 200 000, Rhodia Chemicals). The viscosity of PIB is much more sensitive to temperature than that of PDMS, hence, the viscosity ratio could be varied significantly by changing the temperature of the blends. The viscosities of the pure components are listed in Table I. All components were approximately Newtonian under the experimental conditions. A diblock copolymer of PIB-PDMS was purchased from Polymer Source Inc. Its structural characteristics as measured by the supplier are $M_{w, \text { PIB }}=6150 ; M_{w}$, PDMS $=8000$; polydispersity $\sim 1.3$. The low molecular weights of the blocks imply that there are no entanglements between the blocks and the matrix.

Blends were prepared by mixing the diblock into PIB, and then dispersing this mixture into the PDMS matrix. All mixing was performed by hand with a spatula; since all samples were preconditioned with a specific shear history prior to measurement, differences in mixing conditions may be assumed to be relatively unimportant. This assumption is known to be valid for similar uncompatibilized blends [Vinckier et al. (1996, 
1998)] and was verified for similar compatibilized blends with $p=1.06$ described previously [Velankar et al. (2001b)]. All blends had $10 \mathrm{wt} \%$ of dispersed phase, i.e., volume fraction $\phi=0.108$ at room temperature. Blends are designated $\mathrm{B} x-y$, where $x$ is the viscosity ratio and $y$ is the amount of compatibilizer quoted as a weight percentage of dispersed phase. For example, a " $2 \%$ compatibilized" blend has overall $0.2 \mathrm{wt} \%$ diblock copolymer. In the present work, uncompatibilized, $2 \%$ compatibilized, and $10 \%$ compatibilized blends are investigated; thus the overall wt $\%$ of compatibilizer in the blend was either 0.2 or $1 \mathrm{wt} \%$ of the blend. A 98:2 mixture of PIB:compatibilizer was found to have a viscosity within 5\% that of pure PIB. Similarly, a 98:2 mixture of PDMS:compatibilizer had rheological properties that were indistinguishable from those of pure PDMS. Thus, the compatibilizer did not significantly affect the rheology of the phases even at double the largest concentration used in this research; clearly, the effects discussed in this paper are not attributable to changes in the rheological properties of the bulk phases due to the addition of the compatibilizer.

Dynamic mechanical measurements were performed on a Rheometrics Dynamic Stress Rheometer equipped with a Peltier element to control the temperature, and using a $25 \mathrm{~mm} / 0.1 \mathrm{rad}$ cone and plate geometry. All samples were subjected to preshear of 4.8 $\mathrm{s}^{-1}$ for 3000 strain units. They were then sheared at a lower rate of $1.2 \mathrm{~s}^{-1}$ until steady state was reached. The evolution of morphology was monitored by interrupting the shear flow periodically, and conducting dynamic mechanical measurements at $25 \%$ strain in the frequency range of $0.03-100 \mathrm{rad} / \mathrm{s}$. This relatively large strain value was used to ensure that the torque remained above the limit of sensitivity of the instrument. It was verified that all measurements were in the linear viscoelastic region, and that the morphology did not change during dynamic measurements. Steady-shear viscosities and normal stresses during coalescence were measured with Rheometrics RMS-800 rheometer using $25 \mathrm{~mm} /$ $0.1 \mathrm{rad}$ cone and plate geometry, and a water bath to maintain constant temperature. The strain history for these experiments was identical to that for the dynamic mechanical experiments.

\section{RESULTS}

Here in Sec. III, four rheological properties, the terminal relaxation time, the magnitude of the terminal complex viscosity, the first normal stress difference under steady shear, and the steady-shear viscosity are considered in turn. Only data analysis and experimental observations are described here; discussion is deferred to Sec. IV.

\section{A. Dynamic oscillatory experiments: Terminal relaxation time}

Figure 1 shows typical complex moduli and the magnitude of the complex viscosity for the compatibilized blends studied in this work. The moduli and viscosity of the blends have contributions from bulk phases (here called the "component contribution") and from the interface. The solid lines in Fig. 1 show the component contribution calculated from the Palierne model without interfacial tension (see below for details). In the present case this result is indistinguishable from the volume average contribution of the components (not shown). At high frequencies, the measured moduli and $\left|\eta^{*}\right|$ are nearly identical to the component contribution. The most significant features in Fig. 1 are the shoulder in $G^{\prime}$ and the higher $\left|\eta^{*}\right|$ of the blend at low frequencies compared to the components. The former is discussed next and the latter in Sec. III B.

For blends without a compatibilizer, both these features mentioned above are known to result from the deformation and relaxation of droplets during the dynamic oscillatory experiment [Graebling et al. (1993)]. Dimensional analysis suggests that the relaxation time that corresponds to the shoulder in $G^{\prime}$ is proportional to $\eta_{m} R / \alpha$, with further 


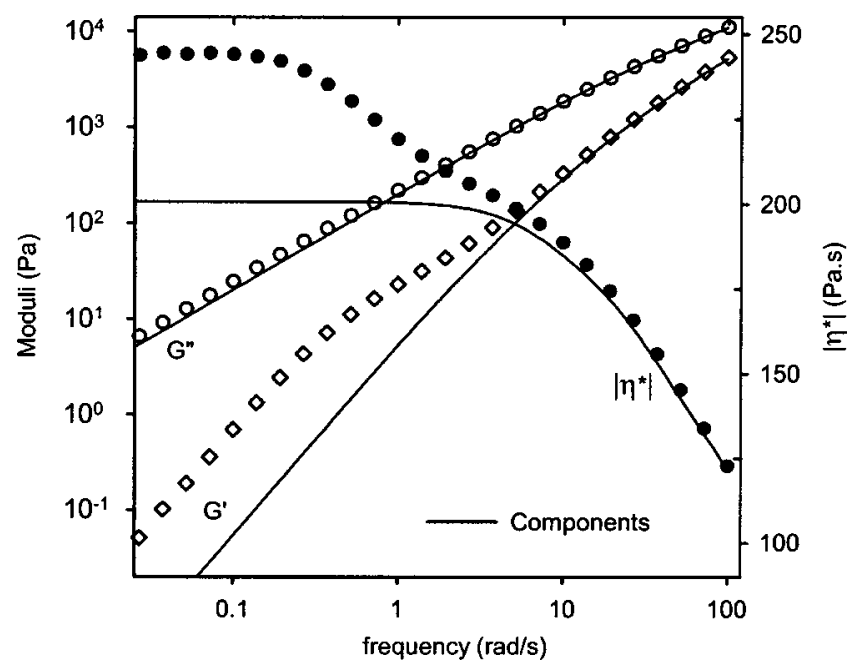

FIG. 1. Complex moduli and magnitude of the complex viscosity for typical compatibilized blends. These data refer to B0.5-2 after shearing at $1.2 \mathrm{~s}^{-1}$ until steady state was reached. Solid lines were calculated using the Palierne model without interfacial tension, and thus represent the contribution expected from the pure components that comprise the blend. This is indistinguishable from the volume average contribution of the components (not shown).

dependence on viscosity ratio $p$ and volume fraction $\phi$. An exact relationship [Eq. (10), presented later] for the relaxation time of blends with small $\phi$ was given in the Palierne model [Palierne (1990); Graebling et al. (1993)]. For uncompatibilized blends, this model relates the complex moduli of blends to those of the components, the volume fraction of the dispersed phase, and the parameter $\alpha / R$. Thus, the mean relaxation time of the blend can be obtained by fitting the Palierne model to the complex moduli of the blend using the quantity $\alpha / R$ as a fitting parameter.

For compatibilized blends, the "complete" Palierne model [Palierne (1990)] predicts more complex behavior due to the interfacial viscoelasticity caused by the presence of the compatibilizer. The main qualitative effect of interfacial viscoelasticity is an additional shoulder in the $G^{\prime}$ vs $\omega$ data. This has been observed experimentally by Riemann et al. (1997) and by Van Hemelrijck et al. (2004). Starting with the complete Palierne model and making some simplifying assumptions, those authors were able fit the model to their data and obtain the viscoelastic properties of the interface. The compatibilized blends studied in this paper, however, like similar blends studied recently [Velankar et al. (2001b)], show moduli that are qualitatively similar to those of uncompatibilized blends, i.e., only a single shoulder is apparent in the $G^{\prime}-\omega$ curves as seen in Fig. 1. A more careful analysis of the relaxation spectrum obtained using the NLREG software. [Honerkamp and Weese (1993)] confirms that the compatibilized blends studied here show only a single relaxation process. Yet, it cannot be concluded that interfacial viscoelasticity is absent. The lack of a second shoulder in the data does not provide conclusive evidence of the absence of interfacial viscoelasticity; Jacobs et al. (1999) showed theoretically that if the interfacial moduli are comparable to the interfacial tension, only a single shoulder is seen in $G^{\prime}$. Indeed, Van Hemelrijck et al. (2004) observed that with increasing amounts of compatibilizer, the two shoulders merged into one. Under such circumstances, it is not possible to obtain reliable values of all the parameters in the "complete" Palierne model; there are simply too many parameters in the complete model 
for them to be obtained by fitting single-shoulder $G^{\prime}$ data such as those shown in Fig. 1. Thus how may be complex moduli of compatibilized blends studied here be analyzed quantitatively?

Three different methods were discussed previously [Velankar et al. (2001b)].

(1) The Palierne model can be used if one simply ignores interfacial viscoelasticity. The rationale is that, since there is only one shoulder in $G^{\prime}$, there is no compelling reason to include interfacial viscoelasticity in the analysis.

(2) The Palierne model can be used still ignoring interfacial viscoelasticity, but including a variable volume fraction of the dispersed phase as an additional fitting parameter. The rationale is that there are some drops with relaxation times that are very far from the mean and they do not contribute to the shoulder in $G^{\prime}$.

(3) The relaxation time of the shoulder can be obtained by fitting the data to a multimode Maxwell model. The Palierne model can be ignored altogether.

For the blends studied here, the first two methods gave poor fits to the data for blends with high viscosity ratios, and so these fits were not used in this paper. All analyses in this paper were performed using the last method [Gramespacher and Meissner (1992)]. Briefly, in this method, the relaxation time $\tau_{d}$ of the shoulder in $G^{\prime}$ was obtained by fitting a multimode Maxwell model to the difference in $G^{\prime}$ of the blends and $G^{\prime}$ expected if the interfacial tension were zero [Dickie (1979); Secor (1992)] (see Supplementary Material in EPAPS Document No. E-JORH02-48-013404. Information on accessing this document is contained at the end of the paper). The quantity $\tau_{d} \dot{\gamma}$, denoted $t^{*}$, is then defined as the dimensionless relaxation time. It should be noted that no specific physical mechanism for the $G^{\prime}$ shoulder is implicit in the calculation of $t^{*}$, i.e., the definition of $t^{*}$ is model independent.

Figure 2 shows the evolution of the dimensionless relaxation time, $t^{*}$, for some selected blends. There are three notable features of the evolution curves. First, $t^{*}$ increases after a step down in shear rate and reaches a plateau after long shearing times. Second, the $t^{*}$ vs shear strain curves shift upward (i.e., towards higher $t^{*}$ ) upon addition of a compatibilizer. Both these features were seen previously for the B1.1 blends [Velankar et al. (2001b)].

A third unusual feature is a maximum in the $t^{*}$ versus shear strain curve for some compatibilized blends. This maximum is prominent for B0.5-10 [Fig. 2(b)] and B0.3-10 (not shown), although weaker maxima can be discerned for some other blends as well. It is not an artifact of data fitting since $G^{\prime}$ at any particular frequency in the terminal region itself goes through a maximum. For blends with $p>0.54$, no maxima could be discerned. Similar maxima have been seen in other compatibilized blend systems studied in this lab [Velankar et al. (2001a)]. The causes of these maxima are not known at present.

The primary concern here in Sec. III A is the effect of added compatibilizer on steady

shear $t_{\mathrm{ss}}^{*}$ (i.e., the value of $t^{*}$ after long shear times) of the blends as a function of the viscosity ratio. For the uncompatibilized blends, Fig. 3 shows $t_{\mathrm{ss}}^{*}$ increases with $p$, gradually below $p=1$, but more steeply as $p$ exceeds 1 . The principal experimental observation in this section is that addition of a compatibilizer has no effect on $t_{\mathrm{ss}}^{*}$ at low $p$, but sharply increases $t_{\mathrm{ss}}^{*}$ at high $p$. This will be discussed further in Sec. IV A.

\section{B. Dynamic oscillatory experiments: Terminal complex viscosity}

The magnitude of the terminal complex viscosity showed virtually no change as coalescence proceeded after decreasing the shear rate from 4.8 to $1.2 \mathrm{~s}^{-1}$. In this section we 




FIG. 2. Evolution of $t^{*}$ during coalescence at $1.2 \mathrm{~s}^{-1}$ for blends with selected viscosity ratios. Similar curves for the B1.1 series of blends have been shown previously [Velankar et al. (2001)].

are concerned with the effect of the compatibilizer on the magnitude of the terminal complex viscosity obtained upon stopping flow after steady-shear flow has been achieved. The viscosity of droplet-matrix emulsions can be made conveniently dimensionless by dividing by the matrix viscosity. The relative terminal complex viscosity $\eta_{0 r}^{*}$ is thus defined as

$$
\eta_{0 r}^{*}=\lim _{\omega \rightarrow 0} \frac{\eta^{*}}{\eta_{m}},
$$




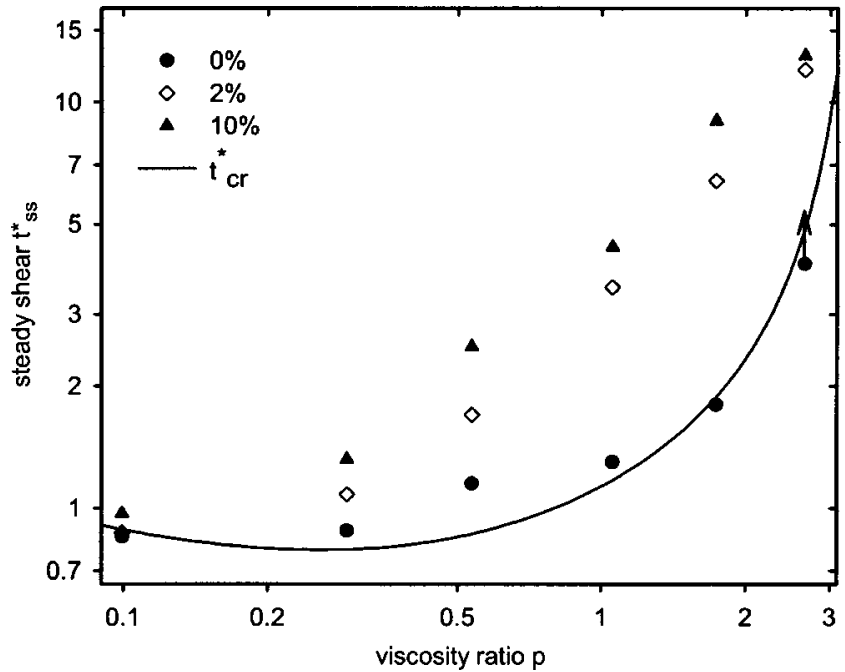

FIG. 3. Dimensionless terminal relaxation times $t_{\mathrm{sS}}^{*}$ after stopping steady shear at $1.2 \mathrm{~s}^{-1}$ as a function of the viscosity ratio. The point corresponding to the B2.7-0 blend is $t^{*}$ after shearing for $\sim 120000$ strain units. The arrow pointing upward indicates that $t^{*}$ was still increasing, and hence the steady state $t^{*}$ for the B2.7-0 blend is higher than the point displayed. The solid line, labeled $t_{\mathrm{cr}}^{*}$, is Eq. (10) with $C a=C a_{\mathrm{cr}}$ substituted from Eq. (11).

where $\eta^{*}$ is the complex viscosity of the blend. The absolute value $\left|\eta_{0 r}^{*}\right|$ is expected to equal $\eta_{0 r}$, the relative steady shear viscosity of the blend in the limit of zero shear rate. Deviations of $\left|\eta_{0 r}^{*}\right|$ from unity reflect the contribution of droplets to the terminal complex viscosity.

The magnitudes of the relative terminal complex viscosities of the uncompatibilized and compatibilized blends are shown in Fig. 4 as a function of the viscosity ratio, $p$. The primary experimental observation is that the compatibilized blends have higher $\left|\eta_{0 r}^{*}\right|$

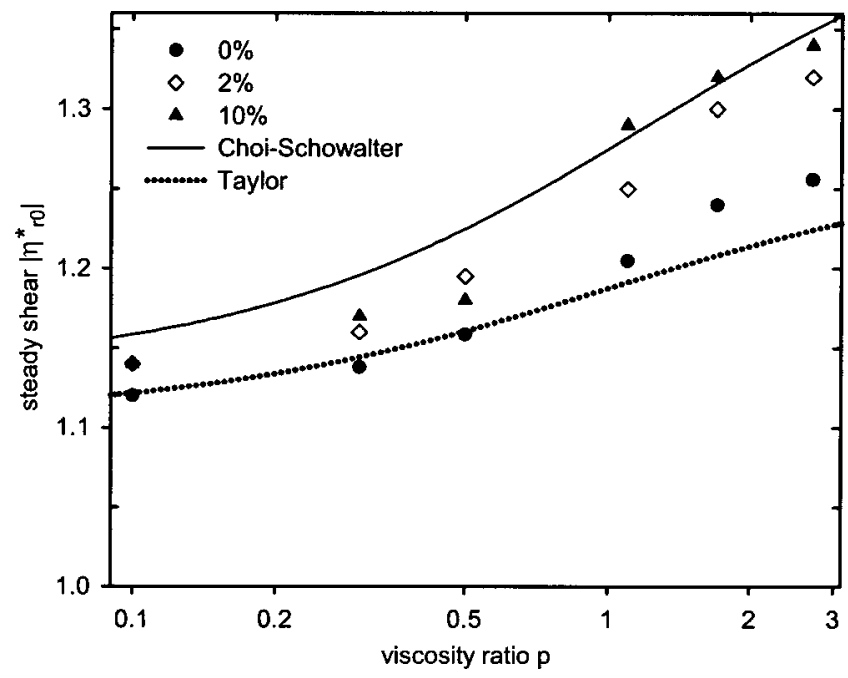

FIG. 4. Magnitude of the relative terminal complex viscosity reached after stopping steady shear at $1.2 \mathrm{~s}^{-1}$. 


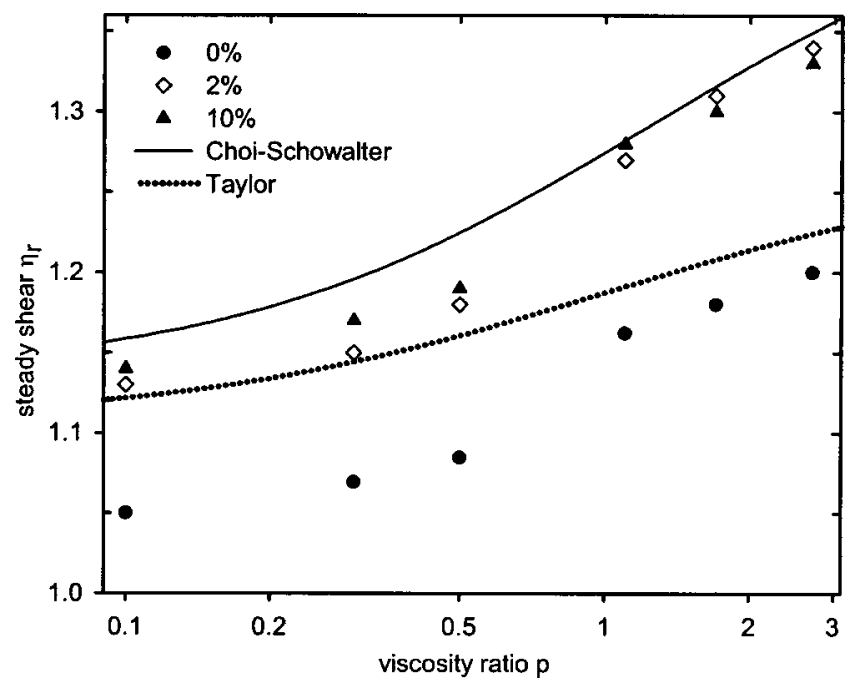

FIG. 5. Relative steady shear viscosity $\eta_{r}$ at $1.2 \mathrm{~s}^{-1}$ for blends.

than uncompatibilized ones, especially at high $p$. Since $\left|\eta_{0 r}^{*}\right|=\eta_{0 r}$, the relative zeroshear viscosity, Fig. 4 also indicates that compatibilized blends are more viscous than uncompatibilized ones in the low shear rate limit.

\section{Steady-shear experiments: Steady-shear viscosity}

The steady-shear viscosity, $\eta$, of all blends decreased slightly during the coalescence process at $1.2 \mathrm{~s}^{-1}$. The present section is concerned with the value of the steady-shear viscosity at $1.2 \mathrm{~s}^{-1}$. As in Sec. III B, the matrix viscosity has been used to render the steady-shear viscosity dimensionless. The relative viscosity,

$$
\eta_{r}=\frac{\eta}{\eta_{m}}
$$

at $1.2 \mathrm{~s}^{-1}$ is shown as a function of $p$ in Fig. 5. Compatibilized blends have a higher relative viscosity than uncompatibilized blends at the same $p$. Moreover, a comparison of Figs. 4 and 5 shows that while $\eta_{r} \approx\left|\eta_{0 r}^{*}\right|$ for compatibilized blends, $\eta_{r}<\left|\eta_{0 r}^{*}\right|$ for uncompatibilized ones, i.e., uncompatibilized blends show shear thinning at $1.2 \mathrm{~s}^{-1}$ whereas compatibilized ones do not.

\section{Steady-shear experiments: First normal stress difference}

For blends with low $p, N_{1}$ was found to increase significantly as coalescence proceeded, whereas for large $p, N_{1}$ was found to change very little after decreasing the shear rate from 4.8 to $1.2 \mathrm{~s}^{-1}$. In all cases, $N_{1}$ reached steady state well before the terminal relaxation time, i.e., $N_{1}$ was found to be far less sensitive to the morphological evolution during coalescence than the linear viscoelastic properties. As in Secs. III A-III C, $N_{1}$ is considered only after reaching steady state at $1.2 \mathrm{~s}^{-1}$ are considered here.

Since the components of the blends studied here are weakly elastic, the bulk phases contribute to $N_{1}$ as well. This component (or bulk) contribution must be subtracted from

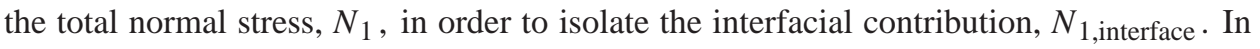




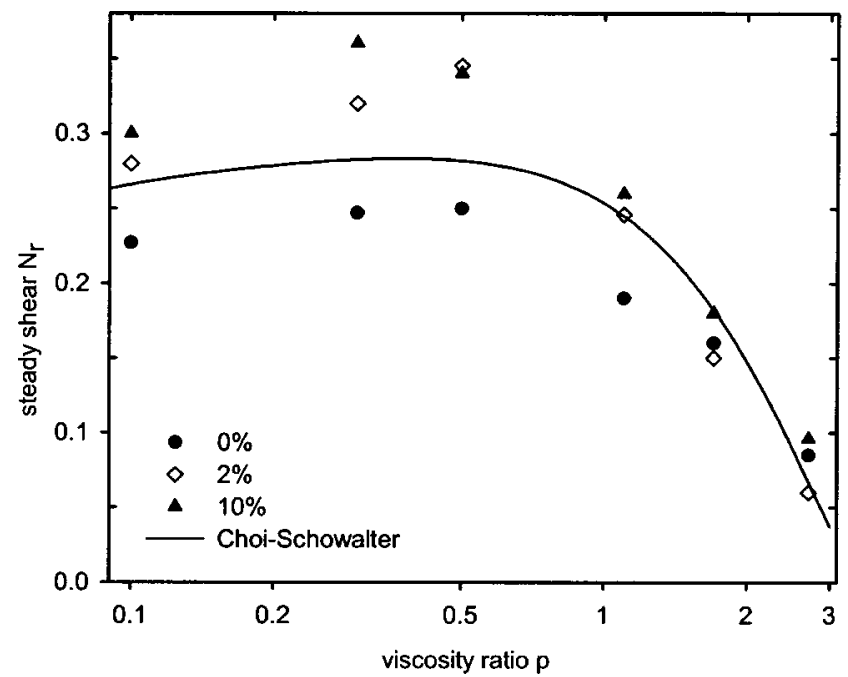

FIG. 6. Relative interfacial normal stress $N_{r}$ under steady shear at $1.2 \mathrm{~s}^{-1}$. The solid line corresponds to the Choi-Schowalter prediction, Eq. (16), with $C a_{\text {cr }}$ from Eq. (11).

past research [Vinckier et al. (1996); Almusallam et al. (2000)], this component contribution has been approximated by a simple volume-weighted average of the components:

$$
N_{1, \text { interface }}=N_{1}-N_{1, \text { components }}=N_{1}-\sum_{k} \phi_{k} N_{1 k} .
$$

While such subtraction of the volume-weighted average of the components does not have a fundamental basis, it has been known to work well for dilute uncompatibilized blends [Jansseune et al. (2001)]. Due to the weak elasticity of the components used here, $N_{1 \text {,components }}$ calculated from Eq. (5) is less than $15 \%$ of the total $N_{1}$, hence, the calculated values of $N_{1 \text {,interface }}$ are not critically dependent on how $N_{1 \text {,components }}$ is calculated.

It has been observed experimentally that under steady shear, $N_{1, \text { interface is proportional }}$ to the shear stress applied [Vinckier et al. (1996); Kitade et al. (1997)], thus, a convenient dimensionless measure of the normal stress due to the interface is the relative interfacial normal stress, $N_{r}$,

$$
N_{r}=\frac{N_{1, \text { interface }}}{\eta_{m} \dot{\gamma}}=\frac{N_{1}-\Sigma_{k} \phi_{k} N_{1 k}}{\eta_{m} \dot{\gamma}} .
$$

Here we are concerned with the effect of the compatibilizer on $N_{r}$. Figure 6 shows $N_{r}$ as a function of the viscosity ratio. At low $p$, addition of the compatibilizer increases $N_{r}$. With an increase of $p, N_{r}$ is reduced more sharply for compatibilized blends than for uncompatibilized ones. Indeed, at the highest viscosity ratio studied, little difference is apparent between the compatibilized and uncompatibilized blends.

In Sec. I we mentioned that the phenomenological question underlying this research is, What is the effect of added compatibilizer on the rheology of immiscible blends? Therefore we summarize the key changes in rheology prior to proceeding with the discussion. Addition of the compatibilizer was found to

(1) increase the terminal relaxation time sharply at high $p$, but not at low $p$;

(2) increase the magnitude of the terminal complex viscosity, especially at high $p$; 
(3) increase the steady-shear viscosity at $1.2 \mathrm{~s}^{-1}$ of blends at all $p$, and reduce their shear-thinning tendency; and

(4) increase the interfacial contribution to the first normal stress at $1.2 \mathrm{~s}^{-1}$ at low $p$, but not at high $p$.

It must be emphasized that none of these results are based on any microscopic model about blend dynamics. The only approximations made in arriving at these results was to highlight the interfacial contribution to $G^{\prime}$ (see the EPAPS material in the reference section) and to $N_{1}$ [Eq. (5)] by subtraction of the component contributions. It must also be noted that all the above observations are apparent even when the amount of compatibilizer is as low as $0.2 \mathrm{wt} \%$ of the blend, and that the results cannot be explained by changes in the rheology of bulk phases due to the compatibilizer.

\section{DISCUSSION}

\section{A. Terminal relaxation time}

Here in Sec. IV A the observation that $t^{*}$ is insensitive to the compatibilizer at low $p$ but increases sharply with compatibilization at high $p$ is considered.

\section{Equivalence between $t^{*}$ and $\mathrm{Ca}$}

First we note that $t^{*}=\tau_{d} \dot{\gamma}$ may be regarded as a kind of capillary number. Dimensional analysis suggests that the relaxation time $\tau_{d}$ of the shoulder must be the ratio of the viscosity that retards interfacial relaxation to the stress that drives interfacial relaxation,

$$
\tau_{d} \propto \frac{\eta_{m}}{\text { interfacial restoring stress }} .
$$

Thus,

$$
t^{*}=\dot{\gamma} \tau_{d} \propto \frac{\dot{\gamma} \eta_{m}}{\text { interfacial restoring stress }} .
$$

The numerator is clearly the hydrodynamic stress that deforms droplets. Thus, being proportional to the ratio of the hydrodynamic stress to the interfacial stress, $t *$ is equivalent to a capillary number as per Eq. (1). For an uncompatibilized blend, this relationship may be made exact since the interfacial stress must scale as $\alpha / R$,

$$
t^{*} \propto \frac{\dot{\gamma} \eta_{m} R}{\alpha}=\frac{\dot{\gamma} \eta_{m} R}{\alpha} f(p, \phi) .
$$

Replacing $\dot{\gamma} \eta_{m} \mathrm{R} / \alpha$ with Ca from Eq. (1), and using the proportionality function $f(p, \phi)$ given by Palierne,

$$
t^{*}=C a \frac{(19 p+16)[2 p+3-2 \phi(p-1)]}{4[10(p+1)-2 \phi(5 p+2)]} .
$$

Clearly, for a particular uncompatibilized blend, $t^{*}$ is simply proportional to the capillary number.

For a compatibilized blend, the interfacial restoring stress is not necessarily $\alpha / R$ since interfacial viscoelasticity can also contribute to the interfacial stress. Nevertheless, the conceptual equivalence between $t^{*}$ and the capillary number as per Eq. (8) still holds. 
Figure 3 shows that the $t_{\mathrm{ss}}^{*}$ after cessation of steady shear increases with compatibilizer at high $p$; therefore in the interpretation here, the steady-shear capillary number increases upon the addition of a compatibilizer at high $p$.

\section{Steady-shear $t_{\mathrm{ss}}^{*} \approx$ critical $t^{*}$}

For uncompatibilized blends, it has been noted previously that after a sufficiently long shear time, the steady-shear drop size is roughly equal to that for breakup [Vinckier et al. (1998); Velankar et al. (2001b)]. This can be tested in our system by comparing $t_{\mathrm{ss}}^{*}$ of the uncompatibilized drops in Fig. 3 with the $t^{*}$ of drops of the critical size for breakup, i.e., substituting $\mathrm{Ca}=\mathrm{Ca} a_{\mathrm{cr}}(p)$ in Eq. (10). Substituting the "Grace curve" equation for $C a_{\mathrm{cr}}(p)$ [Grace (1982); de Bruijn (1989)],

$$
\log \left(C a_{\mathrm{cr}}\right)=-0.506-0.0994 \log (p)+0.124[\log (p)]^{2}-\frac{0.115}{\log (p)-\log (4.08)},
$$

in Eq. (10), the solid line in Fig. 3 is obtained. Clearly, $t^{*} \approx t_{\mathrm{cr}}^{*}$ (i.e., $C a_{\mathrm{ss}} \approx C a_{\mathrm{cr}}$ ) is indeed true for uncompatibilized blends. This can be understood easily: in a shear rate step-down experiment, initially small droplets can grow only until their $\mathrm{Ca}$ is large enough for them to breakup.

If the same is assumed to be true for compatibilized blends, i.e., if we assume that under steady-shear conditions the compatibilized drops also have $\mathrm{Ca}$ equal to their critical value, then Fig. 3 implies that the addition of a compatibilizer increases the critical capillary number and thus stabilizes drops against breakup. Such stabilization of drops due to added surfactant has been reported by direct visual observation previously [Janssen et al. (1994); Van Puyvelde et al. (2002); Hu and Lips (2003)]. Using rheological methods, we drew the same conclusion previously for the B1.1 series of blends [Velankar et al. (2001b)]; the present research however suggests that this stabilization effect is negligible at low $p$, but increases rapidly as $p$ increases.

\section{Mechanism}

A physical mechanism by which the addition of a surface-active compatibilizer can stabilize drops is shown in Fig. 7(c). This picture is derived from past numerical simulations [Stone and Leal (1990); Pawar and Stebe (1996); Li and Pozrikidis (1997); Yon and Pozirikidis (1998); Velankar et al. (2004)] and was elaborated upon in our previous paper [Velankar et al. (2001b)]. Briefly, the flow convects the compatibilizer towards the tips of the drop, causing a gradient in interfacial tension. Corresponding Marangoni stresses tend to reduce internal circulation inside the drops, decrease their deformation, and thus stabilize them. There are two other effects in addition to Marangoni stresses. The first is that the lower interfacial tension at the tips tends to stretch them, thus increasing drop deformation. The second is that the deformed drop has a larger interfacial area than the initially spherical drop, thus resulting in overall dilution of the surfactant; this tends to reduce deformation. Due to the competing effects of dilution and Marangoni stresses on one hand, and lower capillary pressure at the tips on the other, surfactants can stabilize or destabilize drops, depending on parameters such as the viscosity ratio, amount of surfactant, etc. Much numerical research has been devoted to exploring this parameter space [Stone and Leal (1990); Pawar and Stebe (1996); Li and Pozrikidis (1997); Yon and Pozirikidis (1998); Drumright-Clarke and Renardy (2004); Velankar et al. (2004)].

There is considerable experimental evidence that demonstrates that interfacial tension gradients can exist on the surfaces of drops with a compatibilizer. Direct experimental 


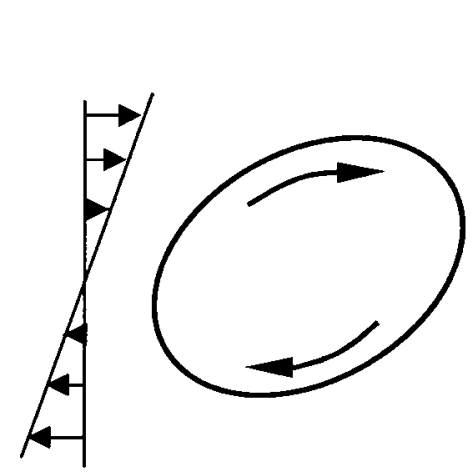

(a)


(d)

FIG. 7. Schematic of droplets in shear flow. (a) Uncompatibilized drops at finite $\mathrm{Ca}$ : interfacial contribution to stress is entirely due to drop deformation. (b) Compatibilized droplet for $\mathrm{Ca} \rightarrow 0$. Bulk flow convects compatibilizer to the ends of the drop. The thickness of the boundary of the drop is indicative of the local compatibilizer concentration. Thin arrows show streamlines; gray arrows show Marangoni stress. Since $\mathrm{Ca}$ $\rightarrow 0$ the droplet is nearly spherical and the interfacial contribution to stress is due to Marangoni stress resulting from redistribution of the compatibilizer on the surface. (c) Compatibilized droplets for finite $\mathrm{Ca}$ : interfacial contribution to stress due to both deformation and comaptibilizer redistribution. Marangoni stress reduces droplet deformation; therefore the drop in (a) is drawn to be more deformed than that in (c). (d) Magnified view of the interface in the dotted circle in (c): Marangoni stress affects the local tangential stress balance at the interface.

evidence of gradients in interfacial tension [Velankar et al. (2001b)] and interfacial concentration [Jeon and Macosko (2003)] has been provided. Indirect evidence in terms of drop shape analysis [Hu et al. (2000); Hu and Lips (2003)], tip streaming [de Bruijn (1993); Janssen et al. (1997); Eggleton et al. (2001)], or an increase in viscosity upon addition of surfactant [Nawab and Mason (1958)] has also been presented. Direct visual evidence that the addition of a compatibilizer makes drops difficult to deform or break has been shown for the B1.1 series of blends previously [Van Puyvelde et al. (2002)].

Here we seek to use this same physical picture to explain the effect of $p$ on $t_{\mathrm{ss}}^{*}$. Following Fig. 7(d), the effect of Marangoni stresses can be qualitatively gauged from a balance of tangential stress at any location on the interface. While this can be done by rigorous stress balance [see Stone and Leal (1990), for example], for the present purposes, an approximate local tangential stress balance is sufficient: 


$$
\eta_{m} \frac{\partial \mathrm{v}_{m}}{\partial z}=\eta_{d} \frac{\partial v_{d}}{\partial z}+\sigma_{\text {Marangoni }}
$$

where $z$ is the direction normal to the surface. This equation suggests that, viewed from matrix phase, the Marangoni stress appears as additional shear stress that has to be overcome in order to maintain shear flow. Therefore, the effects of Marangoni stress are expected to be qualitatively similar to those of an increase in drop viscosity. This analogy is well known from the literature on oil-water emulsions [Lucassen-Reynders and Kuijpers (1992)], and has been shown by numerical simulations [Li and Pozrikidis (1997)]. The data of Fig. 3 agree qualitatively with this analogy: shifting $t_{\mathrm{ss}}^{*}$ for compatibilized blends horizontally to higher $p$ can approximately superimpose them on the $t_{s s}^{*}$ for uncompatibilized blends (not shown, but see the following Sec. IV A 4).

The effect of $p$ on $t_{\mathrm{ss}}^{*}$ is then explained as follows: The preceding section IV A2 noted that $t_{\mathrm{ss}}^{*} \approx t_{\mathrm{cr}}^{*}$; this was verified for uncompatibilized drops and assumed for compatibilized drops. For uncompatibilized drops, the dependence of $t_{\mathrm{cr}}^{*}$ (and therefore of $t_{\mathrm{ss}}^{*}$ ) is shown by the solid curve in Fig. 3. Our analogy says that for compatibilized blends, the additional Marangoni stress is equivalent to a higher $p$. At low $p$ since the solid curve is flat, additional Marangoni stress does not affect $t_{\mathrm{cr}}^{*}$ (nor therefore $t_{\mathrm{ss}}^{*}$ ). At high $p$, since the solid curve increases sharply, the additional Marangoni stress increases $t_{\mathrm{cr}}^{*}$ (and therefore $t_{\mathrm{ss}}^{*}$ ) sharply.

It must be reiterated here that three distinct arguments are made here. First, the conceptual equivalence between $t^{*}$ and the capillary number was noted. Second, it was pointed out that the drops at steady state are at their critical capillary number [verified here and previously by Vinckier et al. (1998) for uncompatibilized drops, assumed for compatibilized drops]. Finally, it was noted that the physical model of Fig. 7(c) predicts that Marangoni stresses are qualitatively equivalent to higher drop viscosity. These three arguments together can explain the effect of the compatibilizer on $t_{\mathrm{ss}}^{*}$.

\section{Possible scaling for Marangoni stress effects}

It is tempting to make the analogy between Marangoni stress and the viscosity ratio more quantitative by attempting to superimpose the $t_{\mathrm{ss}}^{*}-p$ data for samples with different amounts of compatibilizer. A specific superposition scheme,

$$
p_{\text {effective }}=m p\left\{\begin{array}{c}
m>1 \text { for compatibilized blends } \\
m=1 \text { for uncompatibilized blends }
\end{array}\right.
$$

was attempted by Van Puyvelde et al. (2002) to model the coalescence kinetics of compatibilized droplets. Here $m$ is a scaling factor that suggests that addition of compatibilizer is equivalent to a higher effective viscosity ratio. Equation (13) corresponds to a horizontal shift in data along the $\log (p)$ axis. In the viscosity ratio range studied here, Eq. (13) can indeed superimpose the $t_{\mathrm{ss}}^{*}-p$ data and create a reasonable master curve (not shown). Yet, the above scaling is not theoretically justifiable at arbitrary viscosity ratios. In particular, the physical picture in Fig. 7(c) suggests that compatibilized drops with $p$ $=0$ are expected to behave as though they have finite viscosity due to Marangoni stress, a limit that is not captured correctly by Eq. (13). An alternative scaling that does capture the $p=0$ behavior correctly is 


$$
p_{\text {effective }}=\frac{\eta_{d}+\eta_{\text {Marangoni }}}{\eta_{m}}=p+\frac{\eta_{\text {Marangoni }}}{\eta_{m}},
$$

which seeks to model the Marangoni stress as an additive (rather than a multiplicative) contribution to the viscosity of the drop. Thus, in this case, the Marangoni stress is modeled as being proportional to the matrix viscosity, rather than to the viscosity ratio. Unlike Eq. (13), Eq. (14) suggests that the effect of Marangoni stress becomes less important at high $p$. Equation (14) is difficult to test in the present situation where $p$ is varied experimentally by changing both $\eta_{d}$ and $\eta_{m}$.

Clearly, while the analogy between Marangoni stress and the viscosity ratio is appealing intuitively, it is difficult to make it quantitative based only on the present data.

\section{B. Magnitude of the terminal complex viscosity and steady-shear viscosity}

For a dilute emulsion of Newtonian droplets in a Newtonian matrix without a compatibilizer, the relative zero-shear viscosity, $\eta_{0 r}$ (presumed to be equal to the magnitude of the relative terminal complex viscosity $\left.\left|\eta_{0 r}^{*}\right|\right)$ was predicted by Choi and Schowalter (CS) (1975) to be

$$
\eta_{0 r}=\frac{\eta_{0}}{\eta_{m}}=1+\phi \frac{5 p+2}{2(p+1)}+\phi^{2} \frac{5(5 p+2)^{2}}{8(p+1)^{2}} .
$$

Neglecting the last term, i.e., up to first order in $\phi$, this equation is identical to the Taylor prediction [Taylor (1932)] for $\eta_{0 r}$, as well as to Palierne's prediction for $\left|\eta_{0 r}^{*}\right|$ in the absence of interfacial viscoelasticity. Note that for $p \rightarrow \infty$ and $\phi \rightarrow 0$, Eq. (15) reduces to Einstein's equation for the viscosity of a dilute suspension. Both the CS and Taylor predictions are added to Fig. 4 for reference; evidently the Taylor prediction is somewhat closer to the uncompatibilized blend data as was also noted by Grizzuti et al. (2000). Upon increasing shear rates to finite values, theory [Choi and Schowalter (1975)] predicts that drops become oriented along the flow direction, thus reducing the viscosity of the blend. Such shear thinning of uncompatibilized blends has been confirmed experimentally [Vinckier et al. (1996); Grizzuti et al. (2000)].

The concern here in Sec. IV B is the increase in $\left|\eta_{0 r}^{*}\right|$ (or equivalently in $\eta_{0 r}$ ) and in $\eta_{r}$ at finite shear rates with added compatibilizer. All of these quantities have been predicted to increase when an interfacial tension-reducing surfactant is added to an emulsion.

The zero-shear viscosity is considered first. In Oldroyd's picture (1955), a surfactant causes interface dilational viscoelasticity (and sometimes interface shear viscoelasticity as well) which causes the zero-shear viscosity to increase. The same result can be obtained from Palierne's model [Palierne (1990)]. Both Oldroyd as well as Palierne predict that if the interfacial modulus does not decay to zero at low frequencies (elastic interface), the terminal viscosity approaches that of a suspension of hard spheres at the same volume fraction, i.e., the drops behave like solid particles regardless of the viscosity ratio. In the present case, Fig. 4 shows that the relative terminal complex viscosity of compatibilized blends is not independent of the viscosity ratio. Moreover, the blends with low $p$ have $\left|\eta_{0 r}^{*}\right|$ values well below the values expected for suspensions $\left(\left|\eta_{0 r}^{*}\right|=1.27\right.$ according to Einstein's prediction). Clearly, although interfacial viscoelastic effects may indeed be responsible for the increase in terminal viscosity, these effects are not strong enough to make the blends behave like suspensions. This is not surprising; diffusion of the compatibilizer into the bulk must occur slowly, thus purely elastic interfaces are not 
likely. Finally, it should be noted that Nawab and Mason (1958) observed a similar increase in the viscosity of emulsions upon the addition of surfactant. In their case as well, the viscosity remained below that for rigid spheres.

The case of nonzero-shear rates has been addressed by numerical simulations in the limit of low volume fraction of drops by $\mathrm{Li}$ and Pozrikidis (1997) at $p=1$, and by Blawzdziewicz et al. (2000) at all $p$ but small $\mathrm{Ca}$ so that the drops remained nearly spherical. These simulations found that the gradients in interfacial tension along the surface of drops [Fig. 7(c)] contribute to steady-shear stress. In an approximate sense, the Marangoni stress is an additional stress that must be overcome when shearing a compatibilized blend at a given rate, thus giving it a higher viscosity. The simulations predicted that, at low shear rates, $\eta_{r}$ approaches the value predicted by Einstein regardless of the viscosity ratio, in accordance with Oldroyd's prediction. With an increase in shear rate, the contribution of the interfacial tension gradients to the shear stress was reduced, i.e., the compatibilized blends showed shear thinning, yet the surfactant-containing emulsions were predicted to have a higher viscosity than surfactant-free ones at all shear rates. The results of Fig. 5 do indeed show a higher viscosity for compatibilized blends but shear thinning is not evident, at least not at $1.2 \mathrm{~s}^{-1}$. Quantitative comparisons are difficult since the specific properties of the surfactants required for comparison with the simulations are not known for the compatibilizer used here.

Finally, it should be noted that reasonable superposition between compatibilized and uncompatibilized blends of Fig. 4 can be achieved by shifting the compatibilized blend data rightward along the $p$ axis (i.e., compatibilized blends behaving as if their drops had higher viscosity). Thus the results of Fig. 4 are qualitatively consistent with the analogy between Marangoni stress and the viscosity ratio given in Sec. IV A 3. Once again, it is difficult to make this analogy quantitative. Horizontal shifts of the data of Fig. 4 along the $p$ axis according to the scaling in Eq. (13) produce a reasonable master curve (not shown), yet, for the reasons pointed out in Sec. IV A4, Eq. (13) is not justifiable on theoretical grounds at all $p$.

\section{First normal stress difference}

Choi and Schowalter (1975) have predicted $N_{r}$ from the deformation of drops at low shear rates to be

$$
N_{r}=\frac{\phi}{40\left(1+Z^{2}\right)} C a\left[\frac{(19 p+16)}{(p+1)}\left(1+1.25 \phi \frac{5 p+2}{p+1}\right)\right]^{2},
$$

where

$$
Z=\frac{(19 p+16)(2 p+3)}{40(p+1)} C a\left(1+1.25 \phi \frac{19 p+16}{(p+1)(2 p+3)}\right)
$$

Section IV A showed that uncompatibilized blends at steady shear had $C a \approx C a_{\mathrm{cr}}$. Substituting $C a_{\text {cr }}$ from Eq. (11) in Eq. (16), the solid line in Fig. 6 is obtained. Evidently, CS theory, using the critical capillary number, can predict $N_{r}$ well, as was also observed previously [Vinckier et al. (1999)].

We now seek to explain the effect of the compatibilizer on $N_{r}$. Normal stresses in uncompatibilized blends reflect only the deformation and orientation of droplets in the flow as shown in Fig. 7(a). For compatibilized blends however, the interfacial tension gradients can contribute to normal stress as well, i.e., $N_{1 \text {,interface }}$ of compatibilized blends has contributions from both deformation of the interface and from redistribution of the 
compatibilizer on the surface of the droplets [Figs. 7(b) and 7(c)]. Note that in Fig. 7(b), the drop is nearly spherical and normal force is entirely due to interfacial tension gradients; this was the situation simulated by Blawzdziewicz et al. (2000). Thus, for the compatibilized drop in Fig. 7(c), the total normal force from the interface is

$$
N_{r}^{\text {compatiblized }}=N_{r}^{\text {deformation }}+N_{r}^{\text {interfacial tension gradients }},
$$

where the first term on the right corresponds to Fig. 7(a) and the second to Fig. 7(b). Recent numerical simulations [Blawzdziewicz et al. (2000)] suggest that for small deformation, i.e., $C a \ll 1$, the two terms are simply additive, and therefore $N_{r}^{\text {compatiblized }}$ $>N_{r}^{\text {uncompatiblized }}$ is expected at all $p$. However, in reality at finite $\mathrm{Ca}$, the Marangoni stress resulting from interfacial tension gradients tends to reduce drop deformation, i.e., the second term in Eq. (18) tends to reduce the first as shown in Fig. 7(c). Thus, whether $N_{r}$ increases or decreases due to the addition of compatibilizer is determined by the coupling between the two effects shown in Figs. 7(a) and 7(b).

With the physical insight of Fig. 7 and Eq. (18), the trends of Fig. 6 may be understood in qualitative terms. At low $p$, uncompatibilized drops are highly deformable and hence $N_{r}^{\text {deformation }}$ is large; thus, $N_{r}$ is large for uncompatibilized blends at low $p$. In addition, the high deformability at low $p$ implies that interfacial tension gradients are expected to be large for compatibilized blends. Thus, the large value of $N_{r}^{\text {interfacial tension gradients }}$ causes $N_{r}$ to increase with the addition of compatibilizer as seen in Fig. 6. With an increase of $p$ however, even uncompatibilized drops become increasingly difficult to deform, leading to a decrease in $N_{r}$ (solid line in Fig. 6) for the uncompatibilized blends. Additional Marangoni stress at the interface is expected to make compatibilized droplets even less deformable, and hence $N_{r}$ of compatibilized blends is expected to decrease even more rapidly with an increase of $p$. Indeed, Fig. 6 shows that with increasing amounts of compatibilizer, $N_{r}$ decreases more sharply with an increase of $p$; in fact, at the highest value of $p$ studied there is no significant difference between the normal stress of compatibilized and uncompatibilized blends. Yon and Pozrikidis (1998) have indeed noted from numerical simulations that $N_{1}$ becomes independent of the surfactant concentration as the viscosity ratio increases. (It must be remarked, however, that the same simulations note that viscosity also becomes independent of the surfactant as $p$ increases, in contrast with our results.)

Quantitative analysis of these data is not possible since the steady-shear rheological properties depend on both the number and the deformation of droplets, neither of which has been measured in this research.

Finally, it may be noted that the analogy between Marangoni stress and the viscosity ratio definitely fails in this situation: the effect of the compatibilizer on $N_{r}$ cannot be captured by shifting the data for the compatibilized blends along the $p$ axis.

\section{CONCLUSIONS}

The effect of adding a diblock copolymer compatibilizer on the rheological properties of two-phase blends of immiscible polymers was studied for blends with viscosity ratios from 0.1 to 2.65 . Blends had $10 \mathrm{wt} \%$ of dispersed phase and small amounts $(0.2$ or 1 wt $\%$ weight of the blend) of compatibilizer. Linear viscoelastic moduli and steady-shear viscosities and normal stress were measured after a stepdown in shear rate until steadyshear conditions were reached. Addition of the compatibilizer was found to cause the following effects: 
(1) The addition of the compatibilizer does not affect the terminal relaxation time measured after cessation of steady shear at low viscosity ratios, but strongly increases it at high viscosity ratios.

(2) The magnitude of the terminal complex viscosity and the steady-shear viscosity, which reflects the increase in viscosity of the blend due to the presence of droplets, was higher for the compatibilized blends at all viscosity ratios studied. Moreover, compatibilized drops were found to be less shear thinning than uncompatibilized ones.

(3) The relative interfacial normal stress, which reflects the contribution of droplets to the first normal stress difference of the blend in shear flow, was higher for the compatibilized blends at low viscosity ratios. However, with increasing viscosity ratio, the relative interfacial normal stress was found to decrease more sharply for compatibilized blends than for uncompatibilized ones.

The results were interpreted using a physical picture in which flow-induced gradients in interfacial tension, and hence Marangoni stresses, exist on the surface of compatibilized drops. This picture has been established from past experiments and numerical simulations. Most of the observations in this paper are qualitatively consistent with Marangoni stresses stabilizing droplets against breakup in shear flow and contributing to shear and normal stress.

\section{ACKNOWLEDGMENTS}

One of the authors (P.V.P.) is indebted to the FWO-Vlaanderen for a postdoctoral grant. Another author (S.V.) thanks the Research Council of Katholieke Universiteit (KU) Leuven for a postdoctoral fellowship. Financial support from the Research Council (Grant No. GOA 98/06), KU Leuven is gratefully acknowledged.

\section{References}

See EPAPS Document No. E-JORHD2-48-013404 for an Appendix regarding the procedure for fitting $\mathrm{G}^{\prime}$ vs $\omega$ data to obtain the relaxation time $\tau_{d}$. This document may be retrieved via the EPAPS homepage (http:// www.aip.org/pubservers/epaps/html) or from ftp.aip.org in the directory/epaps/. See the EPAPS homepage for more information.

Almusallam, A. S., R. G. Larson, and M. J. Solomon, "A constitutive model for the prediction of ellipsoidal droplet shapes and stresses in immiscible blends," J. Rheol. 44, 1055-1083 (2000).

Blawzdziewicz, J., P. Vlahovska, and M. Loewenberg, "Rheology of a dilute emulsion of surfactant-covered spherical drops," Physica A 276, 50-85 (2000).

Chesters, A. K., "The modeling of coalescence processes in fluid-fluid operations: A review of current understanding," Trans. Inst. Chem. Eng., Part A 69, 259-270 (1991).

Choi, S. J., and W. R. Schowalter, "Rheological properties of non-dilute suspensions of deformable particles," Phys. Fluids 18, 420-427 (1975).

de Bruijn, R. A., "Deformation and breakup of drops in simple shear flows," Ph.D. thesis, Eindhoven University of Technology, Eindhoven, The Netherlands, 1989.

de Bruijn, R. A., "Tipstreaming of drops in simple shear flows," Chem. Eng. Sci. 48, 277-284 (1993).

di Lorenzio, M. L., and M. Frigione, "Compatibilization criteria and procedures for binary blends: A review," J. Polym. Eng. 17, 429-459 (1997).

Dickie, R. A., "Heterogeneous polymer-polymer composites. I. Theory of viscoelastic properties and equivalent mechanical models," J. Appl. Polym. Sci. 17, 45-63 (1973).

Drumright-Clarke, M. A., and Y. Renardy, "The effect of insoluble surfactant at dilute concentration on drop breakup under shear with inertia," Phys. Fluids 16, 14-21 (2004).

Eggleton, C. D., T. M. Tsai, and K. J. Stebe, "Tip streaming from a drop in the presence of surfactants," Phys. Rev. Lett. 87, 048302 (2001). 
Grace, H. P., "Dispersion phenomena in high viscosity immiscible fluid systems and applications of static mixers as dispersion devices," Chem. Eng. Commun. 14, 225-277 (1982).

Graebling, D., R. Muller, and J. F. Palierne, "Linear viscoelastic behavior of some incompatible polymer blends in the melt. Interpretation of data with a model of emulsion of viscoelastic liquids," Macromolecules 26, 320-329 (1993).

Gramespacher, H., and J. Meissner, "Interfacial-tension between polymer melts measured by shear oscillations of their blends," J. Rheol. 36, 1127-1141 (1992).

Grizzuti, N., G. Buonocore, and G. Iorio, "Viscous behavior and mixing rules for an immiscible polymer blend," J. Rheol. 44, 149-164 (2000).

Guido, S., M. Simeone, and M. Villone, "Diffusion effects on the interfacial tension of immiscible polymer blends," Rheol. Acta 38, 287-296 (1999).

Honerkamp, J., and J. Weese, "A nonlinear regularization method for the calculation of relaxation spectra," Rheol. Acta 32, 65-73 (1993).

Hu, Y. T., and A. Lips, "Estimating surfactant surface coverage and decomposing its effect on drop deformation," Phys. Rev. Lett. 91, 044501 (2003).

Hu, Y. T., D. J. Pine, and L. G. Leal, "Drop deformation, breakup, and coalescence with compatibilizer," Phys. Fluids 12, 484-489 (2000).

Iza, M., M. Bousmina, and R. Jerome, "Rheology of compatibilized immiscible viscoelastic polymer blends," Rheol. Acta 40, 10-22 (2001).

Jacobs, U., M. Fahrländer, J. Winterhalter, and C. Friedrich, "Analysis of Palierne's emulsion model in the case of viscoelastic interfacial properties," J. Rheol. 43, 1495-1509 (1999).

Janssen, J. J. M., A. Boon, and W. G. M. Agterof, "Influence of dynamic interfacial properties on droplet breakup in simple-shear flow," AIChE J. 40, 1929-1939 (1994).

Janssen, J. J. M., A. Boon, and W. G. M. Agterof, "Influence of dynamic interfacial properties on droplet breakup in plane hyperbolic flow," AIChE J. 43, 1436-1447 (1997).

Jansseune, T., I. Vinckier, P. Moldenaers, and J. Mewis, "Transient stresses in immiscible model polymer blends during start-up flows," J. Non-Newtonian Fluid Mech. 99, 167-181 (2001).

Jeon, H. K., and C. W. Macosko, "Visualization of block copolymer distribution on a sheared drop," Polymer 44, 5381-5386 (2003).

Kitade, S., A. Ichikawa, N. Imura, Y. Takahashi, and I. Noda, "Rheological properties and domain structures of immiscible polymer blends under steady and oscillatory shear flows," J. Rheol. 41, 1039-1060 (1997).

Koning, C., M. Van Duin, C. Pagnoulle, and R. Jerome, "Strategies for compatibilization of polymer blends," Prog. Polym. Sci. 23, 707-757 (1998).

Li, X., and C. Pozrikidis, "The effect of surfactants on drop deformation and on the rheology of dilute emulsions in Stokes flow," J. Fluid Mech. 341, 165-194 (1997).

Lucassen-Reynders, E. H., and K. A. Kuijpers, "The role of interfacial properties in emulsification," Colloids Surf. 65, 175-184 (1992).

Macosko, C. W., P. Guegan, A. K. Khandpur, A. Nakayama, P. Marechal, and T. Inoue, "Compatibilizers for melt blending: Premade block copolymers," Macromolecules 29, 5590-5598 (1996).

Milner, S. T., and H. Xi, "How copolymers promote mixing of immiscible homopolymers," J. Rheol. 40, $663-687$ (1996).

Nawab, M. A., and S. G. Mason, "Viscosity of dilute emulsions," Trans. Faraday Soc. 54, 1712-1723 (1958).

Oldroyd, J. G., "The effect of interfacial stabilizing films on the elastic and viscous properties of emulsions," Proc. R. Soc. London, Ser. A 232, 567-577 (1955).

Palierne, J. F., "Linear rheology of viscoelastic emulsions with interfacial tension," Rheol. Acta 29, 204-214 (1990).

Pawar, Y., and K. Stebe, "Marangoni effects on drop deformation in an extensional flow: The role of surfactant physical chemistry. 1. Insoluble surfactants," Phys. Fluids 8, 1738-1751 (1996).

Ramic, A. J., J. C. Stehlin, S. D. Hudson, A. M. Jamieson, and I. Manas-Zloczower, "Influence of block copolymer on droplet breakup and coalescence in model immiscible polymer blends," Macromolecules 33, 371-374 (2000).

Riemann, R. E., H. J. Cantow, and C. Friedrich, "Interpretation of a new interface-governed relaxation process in compatibilized polymer blends," Macromolecules 30, 5476-5484 (1997).

Secor, R. B., "Appendix 3A: Curve fitting of the relaxation modulus," in Rheology: Principles, Measurements, and Applications edited by C. W. Macosko (Wiley-VCH, New York, 1994), pp. 127-133.

Stone, H. A., and L. G. Leal, "The effects of surfactants on drop deformation and breakup," J. Fluid Mech. 220, $161-186$ (1990).

Sundararaj, U., and C. W. Macosko, "Drop breakup and coalescence in polymer blends: The effects of concentration and compatibilization," Macromolecules 28, 2647-2657 (1995).

Sung, Y. T., M. S. Han, J. C. Hyun, W. N. Kim, and H. S. Lee, "Rheological properties and interfacial tension of polypropylene poly(styrene-co-acrylonitrile) blend containing compatibilizer," Polymer 44, 1681-1687 (2003). 
Taylor, G. I., "The viscosity of a fluid containing small drops of another fluid," Proc. R. Soc. London, Ser. A 138, 41-48 (1932).

Tucker, C. L., and P. Moldenaers, “Microstructural evolution in polymer blends,” Annu. Rev. Fluid Mech. 34, 177-210 (2002).

Van Hemelrijck, E., P. Van Puyvelde, S. Velankar, C. W. Macosko, and P. Moldenaers, "Interfacial elasticity and coalescence suppression in compatibilized polymer blends," J. Rheol. 48, 143-158 (2004).

Van Puyvelde, P., S. Velankar, and P. Moldenaers, "Rheology and morphology of compatibilized polymer blends," Curr. Opin. Colloid Interface Sci. 6, 457-463 (2001).

Van Puyvelde, P., S. Velankar, J. Mewis, and P. Moldenaers, "Effect of Marangoni stresses on the deformation and coalescence in compatibilized immiscible polymer blends," Polym. Eng. Sci. 42, 1956-1964 (2002).

Velankar, S., P. Van Puyvelde, J. Mewis, and P. Moldenaers, "Effect of compatibilization on the breakup of polymeric drops in shear flow," J. Rheol. 45, 1007-1019 (2001).

Velankar, S., H. Zhou, H. K. Jeon, and C. W. Macosko, "CFD evaluation of drop retraction methods for the measurement of interfacial tension of surfactant-laden drops," J. Colloid Interface Sci. 272, 172-185 (2004).

Velankar, S., Van Hemelrijck, E., Van Puyvelde, P., Moldenaers, P., and Macosko, C. W., "Shear-induced coalescence in compatibilized polymer blends," Proceedings of the 3rd Pacific Rim Conference on Rheology, Vancouver (2001).

Vinckier, I., J. Mewis, and P. Moldenaers, "Relationship between rheology and morphology of model blends in steady shear flow," J. Rheol. 40, 613-632 (1996).

Vinckier, I., M. Minale, J. Mewis, and P. Moldenaers, "Rheology of semi-dilute emulsions: Viscoelastic effects caused by the interfacial tension," Colloids Surf., A 150, 217-228 (1999).

Vinckier, I., P. Moldenaers, A. M. Terracciano, and N. Grizzuti, "Droplet size evolution during coalescence in semiconcentrated model blends," AIChE J. 44, 951-958 (1998).

Yon, S., and C. Pozirikidis, "A finite-element/boundary-element method for flow past interfaces in the presence of surfactants with application to shear flow past a viscous drop," Comput. Fluids 27, 879-902 (1998).

Zarraga, A., M. E. Muñoz, J. J. Pena, and A. Santamaria, "Rheological effects of the incorporation of chlorinated polyethylene compatibilizers in HDPE/PVC blend," Polym. Eng. Sci. 41, 1893-1902 (2001). 